\title{
Clonal origin and spread of metastatic prostate cancer
}

\author{
Jamie L Van Etten ${ }^{1}$ and Scott M Dehm ${ }^{1,2,3}$ \\ ${ }^{1}$ Masonic Cancer Center, University of Minnesota, Minneapolis, MN, USA \\ 2Department of Laboratory Medicine and Pathology, University of Minnesota, Minneapolis, MN, USA \\ 3Department of Urology, University of Minnesota, Minneapolis, MN, USA
}

Correspondence should be addressed to S M Dehm

Email

dehm@umn.edu

\begin{abstract}
Metastatic disease is responsible for the majority of prostate cancer deaths. The standard treatment for metastatic disease is surgical or chemical castration in the form of androgen deprivation therapy. Despite initial success and disease regression, resistance to therapy ultimately develops and the disease transitions to castrationresistant prostate cancer, which is uniformly fatal. Thus, developing an understanding of genetic evolution in metastasis and in response to therapy has been a focus of recent studies. Large-scale sequencing studies have provided an expansive catalog of the mutation events that occur in the prostate cancer genome at various stages of disease progression. Small-scale studies have interrogated the genomic composition of multiple metastatic sites within individual patients or have tracked clonal evolution longitudinally in tissues, circulating tumor cells, or circulating tumor DNA. Collectively, these efforts have provided a new conceptual framework for understanding the origin of prostate cancer, as well as the origin and evolution of metastatic disease. In this review, we highlight these recent insights into the spatiotemporal landscape of genetic evolution of prostate cancer.
\end{abstract}

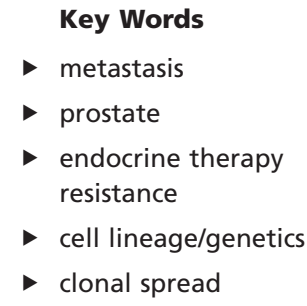

Endocrine-Related Cancer (2016) 23, R207-R217

\section{Introduction}

Prostate cancer is the most frequently diagnosed cancer in men and accounts for an estimated 142,000 deaths in eveloped countries each year (Torre et al. 2015). Fiveyear survival rates for localized diseases are high (>90\%) because localized cancers can be treated successfully with surgery and radiation. In normal prostate cells, the androgen receptor (AR) functions as a master transcriptional regulator activated by the androgens testosterone and dihydrotestosterone. Accordingly, prostate cancer presents as an androgen and AR-dependent disease (Dehm \& Tindall 2011). For patients in whom surgery and radiation are not curative, the standard systemic treatment is functional suppression of $A R$ transcriptional activity through androgen deprivation therapy (ADT). ADT includes surgical castration, pharmacological castration, and antiandrogen therapy (Dehm \& Tindall 2011). ADT is initially successful in most patients; however, over time, castration-resistant disease develops. Therefore, development of therapy resistance and transition to castration-resistant prostate cancer (CRPC) are major clinical challenges. CRPC is characterized by rising PSA levels (signifying reactivation of AR transcriptional activity), an increase in tumor size, and metastatic spread (Knudsen \& Scher 2009). Secondline ADT drugs that provide a more effective blockade of systemic androgen synthesis (abiraterone acetate) or act as higher-affinity antagonists of AR (enzalutamide) improve overall survival (Beer et al. 2014, Ryan et al. 2013).

Published by Bioscientifica Ltd 
However, in many patients, second-line ADT fails to achieve control of tumor growth, which indicates frequent occurrence of primary resistance. Additionally, secondary resistance to these therapies inevitably develops in a relatively short time frame and CRPC thus remains a uniformly fatal disease.

Clonal populations of cancer cells are in persistent evolution in response to environmental conditions. The concept of tumor evolution was proposed by Nowell in 1976 based on cytogenetic data (Nowell 1976). In this early model, a cell of origin acquires genetic alterations that promote neoplasia. Further genetic instability fuels clonal expansion of "fit" clones that ultimately leads to advanced malignancy, metastasis, and emergence of therapy resistance (Nowell 1976). Large-scale genome sequencing studies in recent years have provided expansive catalogs of the genomic aberrations in primary and advanced prostate cancers and have offered insight into deregulated molecular pathways at each stage of disease for the development of novel targeted therapies (Abeshouse et al. 2015, Robinson et al. 2015). However, these large-scale genome sequencing studies have focused on single samples of bulk tumors collected at a single time point from individual patients, which does not enable a complete understanding of tumor heterogeneity. An additional limitation with this single-site/single-time sampling approach is that it does not reveal the subclonal changes that occur within tumor populations spanning disease progression and recurrence after therapeutic intervention.

More recently, small-scale studies focused on sampling of multiple metastatic sites or longitudinal sample collection combined with computational reconstructions of clonal evolution have confirmed that primary tumors and adjacent normal tissue consist of multiple clonal populations. These studies have further revealed that metastatic spread can occur through monoclonal or polyclonal seeding between metastases or in waves originating from the primary tumor. Moreover, genomic alterations associated with resistance to ADT have been identified, including AR and AR pathway components (further expanded upon and reviewed in Watson et al. (2015)), highlighting an opportunity for the development of biomarkers representative of resistant subclones vis-a-vis personalized medicine approaches. In this review, we discuss the recent efforts to understand clonal evolution and map the clonal spread and expansion of metastatic prostate cancer. Collectively, these studies have illuminated several intricate mechanisms by which discrete clones undergo selection and spread in individual patients.

\section{Genomic approaches to interrogate clonal framework in tumor tissues}

The multifocal and heterogeneous nature of prostate cancer can hinder efforts to understand tumor cell clonality, particularly in metastatic disease. Until recently, the tools available to assess clonality within multifocal and heterogeneous tumor samples relied upon histological assessment, cytogenetic approaches such as FISH, and molecular approaches such as PCR. Many malignancies exhibit multiclonality including AML, breast, melanoma, esophageal, and non-small-cell lung cancer (Merlo et al. 2010, Ding et al. 2012, Nik-Zainal et al. 2012, Bolli et al. 2014, McFadden et al. 2014, Rashid et al. 2014). By applying the concept of population genetics of the most recent common ancestor, it has been possible to quantify genomic aberrations and thereby define clonal and subclonal populations of cells responsible for metastatic seeding and evasion of therapy (Campbell et al. 2008, Nik-Zainal et al. 2012). The first step in this strategy is to determine tumor purity, which is critical because resected tumors and biopsied tumor tissues harbor stromal cell infiltrates. This can be accomplished by identifying the fraction of tumor cells carrying clusters of mutations relative to normal tissue. (Van Loo et al. 2010). To define clonal and subclonal populations of cells within metastases at different body sites, mutant allelic fraction in multiple tumor sections relative to normal tissues can be calculated, taking into account tumor purity and copy number (Campbell et al. 2008, Nik-Zainal et al. 2012). Any mutations present at a smaller proportion in comparison with the clonal population would be indicative of subclonal events.

Using these methods, clonal evolution can be tracked across various disease states for which there is tissue available and can be used to reconstruct a spatiotemporal mutational landscape of disease progression. For example, this approach was used to retrospectively construct a time line of clonal evolution and metastatic spread in a rapid autopsy study of ten subjects that died from prostate cancer (Gundem et al. 2015). Alternatively, these methods enable monitoring of clonal evolution in real time. For example, one study collected longitudinal samples of blood and tumor biopsies from four patients with advanced prostate cancer as their disease progressed from localized prostate cancer, to biochemical recurrence, and ultimately to CRPC (Hong et al. 2015). A separate study examined clonal evolution in response to selective pressures of therapy by targeted sequencing of plasma DNA and targeted deep sequencing of tumor biopsies obtained from patients

Published by Bioscientifica Ltd. 
with ERG-positive cancers (Carreira et al. 2014). Together, these studies build upon an existing catalog of known mutations, structural alterations, and altered pathways to inform our understanding of the clonal evolution and spread of prostate cancer, particularly in response to AR-targeted therapies.

\section{Primary prostate cancer is multifocal and heterogeneous}

Prostate cancer often presents as discrete foci within the prostate capsule (Ruijter et al. 1999). Whether these discrete foci represent independent clones or geographically separated, yet related, clonal populations has been a subject of debate (Cheng et al. 1998, Barry et al. 2007, Kobayashi et al. 2008, Boyd et al. 2012, Lindberg et al. 2013, Boutros et al. 2015, Cooper et al. 2015). For example, in a study of 254 prostatectomy specimens, nearly half harbored multiple individual tumor foci separated by at least $3 \mathrm{~mm}$ within the resected gland (Villers et al. 1992). In these cases, it is possible that two distinct tumor cell populations arose in the prostate. Alternatively, independent foci may represent related clonal populations. Similarly, a study of
17 radical prostatectomy specimens indicated the presence of multiple tumor foci in several samples. Analysis of discrete foci revealed concordant as well as discordant allelic imbalances, suggesting variability in clonal origin of tumor foci within an individual prostate gland (Ruijter et al. 1999). In a separate study of 47 prostatectomy specimens, $\sim 20 \%$ were multifocal and exhibited varying grades of disease. Loss of heterozygosity (LOH) was observed within tumors; however, intertumoral LOH was not predictable from intratumoral patterns of allelic loss (Hugel \& Wernert 1999).

Primary prostate cancer can be subclassified according to a recurrent set of mutually exclusive genomic alterations that occur early in disease development (Abeshouse et al. 2015). Specifically, analysis of 333 primary prostate carcinomas in The Cancer Genome Atlas (TCGA) project identified seven mutually exclusive genetic subtypes including ERG, ETV1, ETV4, FLI1, SPOP, FOXA1, and IDH1 (Abeshouse et al. 2015). Roughly 75\% of prostate cancers fell into one of these genetic subtypes; however, $\sim 25 \%$ remained uncharacterized (Fig. 1). Identification of additional genetic alterations has provided evidence that primary tumors exhibit multiclonality coincident

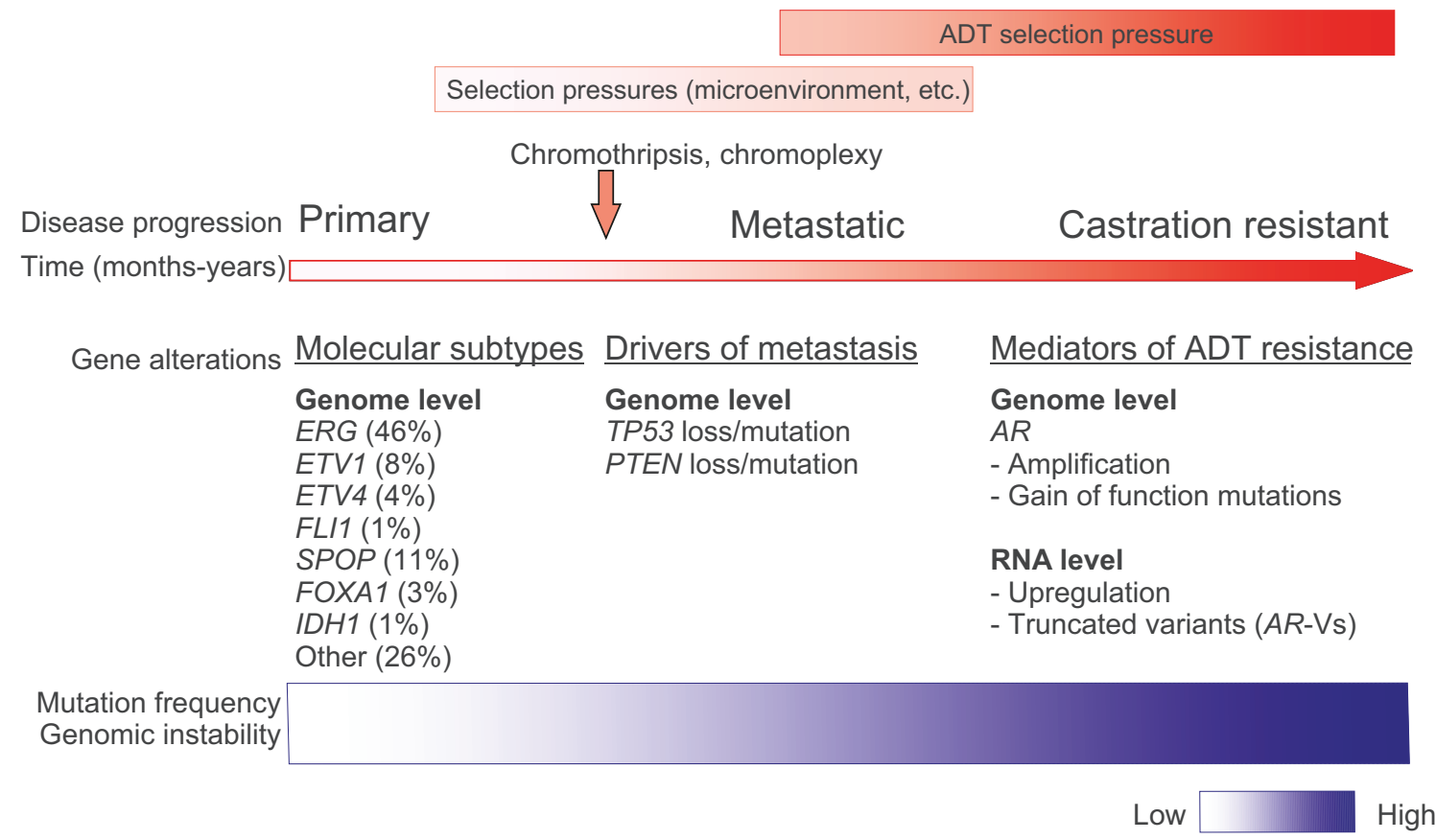

\section{Figure 1}

Schematic of common gene and pathway alterations across prostate cancer disease states, as identified by computational reconstructions of tumor evolution. Environmental factors impart selection pressures on clonal populations to drive metastasis and, ultimately, resistance to ADT. The TCGA study identified seven mutually exclusive genetic subtypes, listed here by percentage of tumors (333 total) (Abeshouse et al. 2015 ). TP53 and PTEN inactivation are observed at higher frequencies in metastatic CRPC versus primary prostate cancer and are thought to play driver roles in metastasis (Abeshouse et al. 2015). Mediators of ADT resistance were observed to occur after metastatic spread (Carreira et al. 2014, Gundem et al. 2015, Hong et al. 2015, Romanel et al. 2015) and have been further reviewed in Watson et al. 2015. 
with multifocal disease. For example, whole-genome sequencing (WGS) in a cohort of five patients (Gleason scores 7-8) who underwent radical prostatectomies provided the first WGS study demonstrating extensive genomic heterogeneity within prostate tumors (Boutros et al. 2015). One example of multiclonality within the resected prostate was a patient whose tumor was dissected into four regions, each of which harbored disease foci. The index lesion shared SPOP mutations and chromosomal deletions (on chr8, 16) with one focus, but not the remaining two dissected foci, which shared a deletion on chr19 (Boutros et al. 2015). Importantly, this study also discovered a previously unidentified recurrent amplification of MYCL associated with TP53 loss (Boutros et al. 2015). Similarly, morphologically normal tissues have been found to exhibit high levels of mutations and distinct $E R G$ fusions that are present in malignant tissues (Cooper et al. 2015). In many cases, discrete tumor foci within the prostate comprised of distinct clones representing different genetic subtypes or harboring different driver mutations, indicating independent clonal origins. For example, interrogation of two independent foci present in a prostatectomy specimen demonstrated that they were distinct in clonal origin: one was ERG positive, whereas the other exhibited SPOP mutation (Barbieri et al. 2012). It is important to note, however, that in this cohort (112 patients), the authors reported only this single case of multifocal disease. In a smaller study of four patients, three discrete prostate cancer foci had no common somatic ancestor as assessed by high-frequency single-nucleotide variants (SNVs) and copy number alteration profiling (Lindberg et al. 2013). In a study of 48 patients with ERG-positive prostate cancer, areas of high-grade prostatic intraepithelial neoplasia (PIN) and intraductal carcinoma proximal to areas of invasive carcinoma were found to be ERG positive (Haffner et al. 2015). High-resolution studies of tissues from seven of those patients demonstrated that subclonal PTEN loss present in the ERG-positive invasive adenocarcinoma was also present in adjacent PIN lesions, indicating retrograde spreading to benign structures (Haffner et al. 2015).

Gradual acquisition of genomic alterations is considered to be the primary driver of tumorigenesis. However, accelerated mechanisms of genomic alteration, such as chromothripsis, a massive and catastrophic reshuffling of entire chromosomes or regions of chromosomes, and chromoplexy, complex rearrangements that may arise from multiple rounds of DNA repair, could account for shortened time frames of tumorigenesis and metastasis (Stephens et al. 2011, Baca et al. 2013). Indeed, chromoplexy has been shown to capture multiple genetic hits in one structural rearrangement event, which would be predicted to accelerate tumorigenesis and clonal evolution. For example, in one case of prostate cancer, the coding regions of several tumor suppressor genes, including ETV6, ETV3, and $C D K N 1 B$, were disrupted by a single chromoplexy event involving 25 rearrangements spanning three chromosomes (Baca et al. 2013). In another case of prostate cancer, 27 rearrangements occurred in a single chromoplexy event to yield an SMAD rearrangement as well as an oncogenic TMPRSS2:ERG fusion (Baca et al. 2013).

The most common lesions in primary tumors, affecting $\sim 50 \%$ of cancers, are ETS fusions, in which AR-regulated or otherwise highly active promoters and/or enhancers such as TMPRSS2 are fused to the coding regions of ERG, ETV1, ETV4, or FLI1 (Taylor et al. 2010, Barbieri et al. 2013, Abeshouse et al. 2015, Adamo \& Ladomery 2015). Interestingly, these gene fusion events have been shown to be promoted by androgen/AR-regulated changes in intra-nuclear chromatin organization (Lin et al. 2009, Mani et al. 2009, Weischenfeldt et al. 2013). For example, AR binding sites have been shown to exist near TMPRSS2, $E R G$, and ETV1 fusion break points, and androgeninduced binding of AR to these sites has been shown to bring $5^{\prime}$ and $3^{\prime}$ gene fusion partners into close proximity (Lin et al. 2009). Genotoxic stress has been shown to cooperate with the androgen-mediated proximity effect to induce TMPRSS2:ETS or TMPRSS2:ETV1 gene fusion events similar to those observed in clinical prostate cancer (Lin et al. 2009, Mani et al. 2009). Interestingly, this androgenmediated proximity effect may promote the genesis of additional rearrangement events in the prostate cancer genome, as AR binding sites are frequently found adjacent to rearrangement break points (Weischenfeldt et al. 2013).

\section{Clonal evolution and spread of metastatic prostate cancer}

Relative to metastatic disease, primary tumors exhibit low mutation frequencies and genome instability (Abeshouse et al. 2015, Robinson et al. 2015). As the disease progresses to the metastatic and castration-resistant phases, extensive mutations, structural rearrangements, and genome instability are observed. These genomic alterations are indicative of clonal evolution and subclonal selection to survive environmental pressures. Importantly, altered AR signaling after metastatic spread spans subtypes occurs in more than $50 \%$ of metastases and remains a key target for therapies (Robinson et al. 2015). Commonly identified genomic alterations in metastatic CRPC are TP53 mutation or loss,

Published by Bioscientifica Ltd 
PTEN loss, PI3K pathway defects, DNA repair pathway deficiencies, and amplification or mutation of AR (Fig. 1) (Kim et al. 2007, Grasso et al. 2012, Lohr et al. 2014, Hong et al. 2015, Robinson et al. 2015). It has been challenging to differentiate alterations that promote metastasis vs alterations that promote therapy resistance because, historically, studies of metastatic prostate cancer have been performed using tissues from heavily treated CRPC patients. Exome sequencing of CRPC metastases obtained at rapid autopsy from a cohort of 50 patients identified frequent mutations in TP53, AR, FOXA1, PTEN, and among others (Grasso et al. 2012). Indeed, the finding that mutation or amplification of $A R$ does not occur in primary prostate cancer but is a common genomic alteration in CRPC metastases appears to implicate AR as a driver of metastasis (Abeshouse et al. 2015). However, more detailed wholegenome sequencing studies have indicated AR as a driver of therapy resistance, rather than a driver of metastasis (Fig. 1) (Carreira et al. 2014, Gundem et al. 2015, Hong et al. 2015, Romanel et al. 2015). For instance, interrogation of subclonal structure within CRPC metastases demonstrated that different $A R$ gene alterations can occur in different metastatic foci within a single patient, indicating that these alterations occurred in response to therapy, after metastatic spread (Gundem et al. 2015). However, this same analysis indicated that TP53 loss, DNA repair pathway alterations, and PTEN loss occurred before metastatic spread (Gundem et al. 2015). Further, the frequencies of TP53 and PTEN inactivation were higher in metastatic CRPC compared with primary prostate cancer, indicating driver roles in metastasis (Abeshouse et al. 2015).

\section{Clonal spread between the primary tumor and distant metastatic sites is multidirectional}

Metastases are considered to be monoclonal in origin with respect to the primary tumor, with clones sharing a number of common mutations but exhibiting divergent somatic mutations between different metastatic sites (Gundem et al. 2015, Hong et al. 2015). Early rapid autopsy investigations observed that PSA immunostaining varied across and within metastatic foci, suggesting that multiple subclonal populations existed within metastases (Roudier et al. 2003, Shah et al. 2004). Genome-wide copy number analysis and targeted sequencing studies revealed that metastases within a patient shared common mutations and therefore shared clonal origins (Liu et al. 2009, Robbins et al. 2011). However, these same studies also demonstrated that these tumors harbored sets of divergent mutations, indicating that subclonality existed between individual tumor foci. For example, analysis of three spatially distinct metastases from a single autopsy subject revealed shared amplifications at chromosomes $5 p$ and $14 q$ as well as a set of shared somatic mutations (Robbins et al. 2011). The presence of common copy number alterations and somatic mutations across multiple metastases indicated a clonal progenitor cell, presumably from the primary tumor. However, in this study, primary tumor tissue was not available for this patient (Robbins et al. 2011). Similarly, copy number analysis and targeted resequencing of 80 anatomically distinct metastatic foci isolated from 24 patients with CRPC indicated that metastases shared a clonal origin in most patients (Liu et al. 2009). Importantly, metastases shared clonal copy number variations with the primary tumor in five patients with available tissues (Liu et al. 2009). These observations indicate that prostate cancer metastases originate from a common clonal progenitor in the prostate. However, as discussed below, alternate explanations may also be possible in light of recent data, demonstrating that cells from prostate cancer metastases may be able to reseed the surgical bed where the original primary tumor existed (Liu et al. 2009, Hong et al. 2015).

A commonly accepted model of metastasis depicts subclonal populations colonizing metastatic sites in waves originating from the primary tumor (Wan et al. 2013). In this model, subclonal populations within the primary tumor compete for dominance and are selected for survival and growth by environmental selection pressures (Greaves $\&$ Maley 2012). In support of this model, a longitudinal study of four patients demonstrated that metastases were seeded in temporally separated waves originating from the primary tumor (Hong et al. 2015). Presumably, as the primary tumor acquired new mutations, structural variations, and copy number alterations, new metastatic subclonal populations were released to seed and reseed organ sites (Hong et al. 2015) (Fig. 2A). Similarly, in a single case study, anatomically distinct metastatic sites from a patient with CRPC were found to share many genetic alterations including high-level amplification of the $A R$ locus, PTEN loss, TP53 loss, and mutation of SPOP. As a result of these shared genomic alterations, these clones were interpreted to be monoclonal in origin (Haffner et al. 2013). Interestingly, examination of the microdissected primary tumor revealed that a small $2.2 \times 1.3 \mathrm{~mm}$ welldifferentiated Gleason pattern 3 lesion exhibited PTENnegative immunohistological staining. DNA sequencing of this lesion revealed the same 4 base pair deletion in PTEN observed in the metastatic clones concurrent with TP53 mutation, suggesting that this lesion harbored the

Published by Bioscientifica Ltd 
A

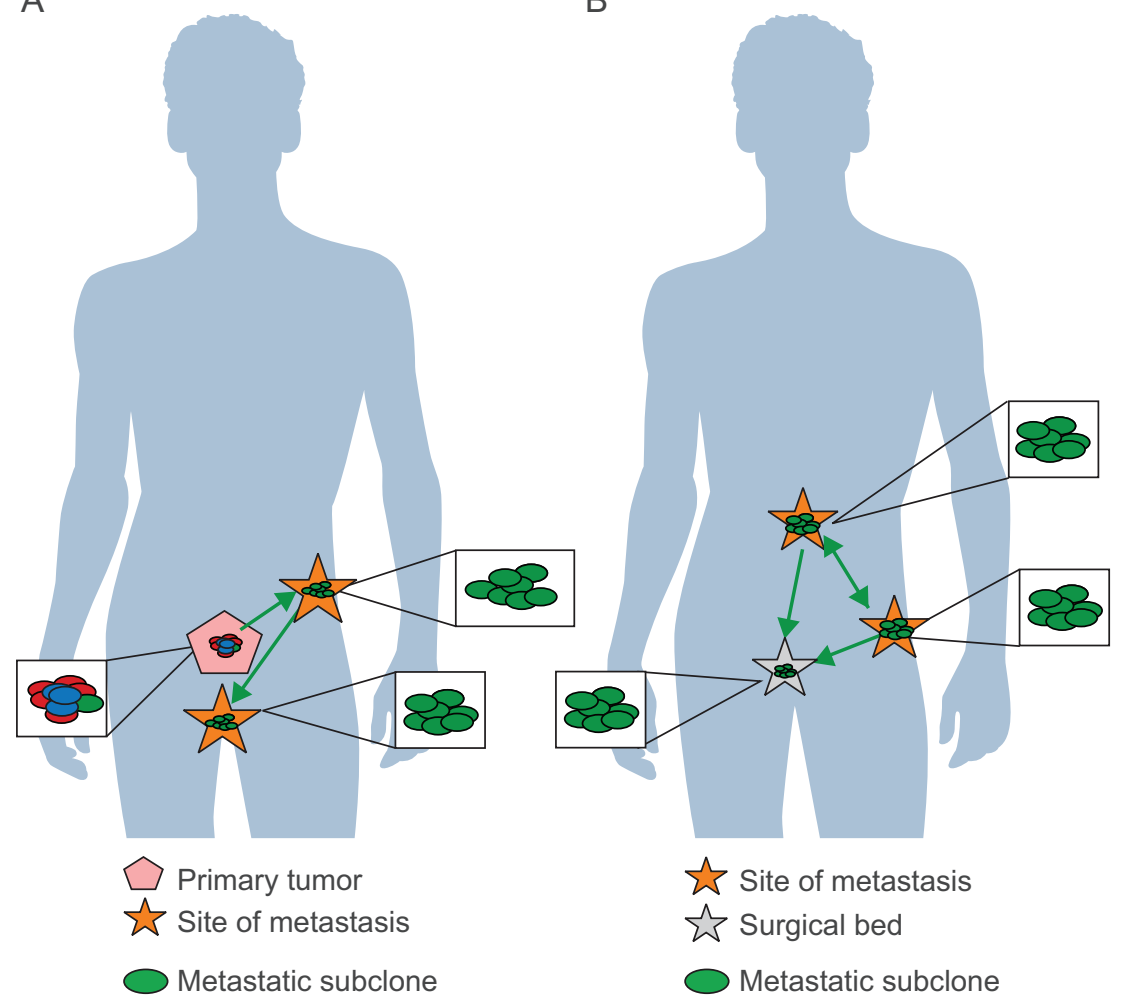

C

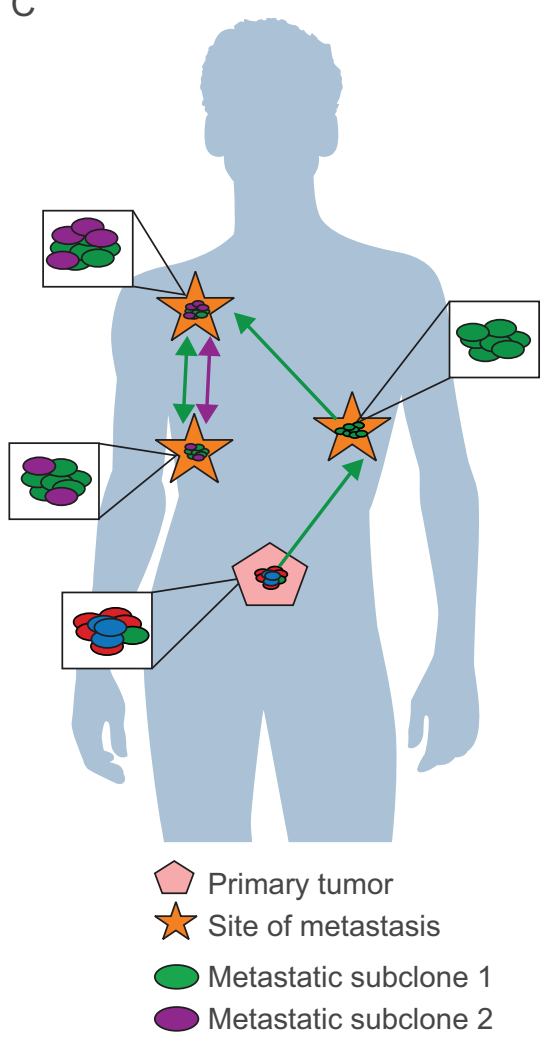

\section{Figure 2}

(A) A rare clone (green, "metastatic subclone") within the primary tumor acquires metastatic potential and seeds distinct metastatic sites in temporal waves. Clonal populations originating in the primary tumor can seed sequential anatomic sites such as the pubis and penis in a temporally distinct fashion (depicted by green arrows) (Hong et al. 2015). (B) Metastatic clones (green, "metastatic subclone") that seeded multiple sites (depicted by green arrows), including the sacrum and iliac crest in a bidirectional manner, can also reseed the primary surgical bed (Hong et al. 2015). (C) Clonal populations from metastatic sites seed anatomically distinct sites of metastasis in a polyclonal fashion. Here, a hypothetical metastatic clonal population (green, "metastatic subclone") seeds the left rib and shoulder (depicted by green arrows). A subclonal population arises in the shoulder (purple, "metastatic subclone 2"), and together, these populations colonize the rib (adapted from Gundem et al. 2015).

progenitor cell that seeded distant metastases (Haffner et al. 2013). Surprisingly, the surrounding higher grade Gleason pattern 4 tumor tissue did not harbor the same underlying mutations as the metastases. These data indicate that the primary index lesion does not always harbor the lethal clone that is ultimately responsible for seeding distant metastasis in the patient.

Another common mechanism of metastasis in advanced prostate cancer is via seeding of de novo metastatic sites by cells originating from distant metastasis (Gundem et al. 2015) (Fig. 2B). Furthermore, "local recurrences" after surgery may also arise via a similar mechanism of cells from distant metastases seeding the prostatic cavity after radical prostatectomy (Hong et al. 2015) (Fig. 2B). The mechanistic underpinnings of metastasis-to-metastasis and metastasis-to-primary site seeding remain to be clarified.

\section{Multidirectional spread can lead to polyclonal seeding of metastatic sites}

Similar to primary prostate cancer, a common model to explain intratumoral heterogeneity at metastatic foci has been that cells acquiring new genetic alterations are under constant environmental and therapeutic selection pressure, leading to continuous expansion and contraction of tumor subclones. However, in addition to this monoclonal model of metastatic evolution and spread, a polyclonal pattern has also been described. For example, Gundem and colleagues assessed the clonal relationship between subclones occupying different metastatic sites. On a phylogenetic tree, a truncal mutation in a pair of samples would be apparent if the same mutation was present in $100 \%$ in the cancer cell fraction at two different metastatic sites. Conversely, non-truncal (or branch) mutations would be apparent if the same mutation was present in less than

Published by Bioscientifica Ltd. 
$100 \%$ of the cancer cell fraction at two different metastatic sites. Using this logic, it was found that $50 \%$ of the subjects in a rapid autopsy study exhibited polyclonal seeding of multiple metastatic sites (Gundem et al. 2015). Polyclonal seeding was defined in this study as multiple genetically distinct subclones colonizing a single metastatic site (Fig. 2C). Interestingly, all instances of polyclonal seeding involved mutations in genes with known involvement in therapy resistance, indicating that polyclonal seeding and interclonal cooperation may be required to evade therapy.

Overall, these studies have illuminated details of the complex mechanisms underlying metastatic seeding. This process can be initiated by monoclonal or polyclonal populations originating in both the primary tumor and metastases in other sites (Gundem et al. 2015, Hong et al. 2015). Polyclonal populations involved in these seeding events were more similar to one another than to clones occupying other metastatic sites (Gundem et al. 2015). Whether that is due to proximity effects on subclonal evolution or, conversely, characteristics of the metastatic niche that impart habitability remains to be tested. Importantly, it has become clear from these collective data that multiple mechanisms of seeding give rise to metastases in different body sites, highlighting the need for personalized medicine via monitoring of genetic changes in tumor cells within patients over the course of their disease and stages of treatment.

\section{Subclonal expansion to escape targeted therapy}

Treatment with AR-targeted ADT imparts a selection pressure upon tumor foci (Fig. 3). Clones harboring gene alterations that promote ADT resistance, such as $A R$ point mutations, alterations in AR pathway components, and alterations in MYC and CTNNB1, were shown to seed and reseed multiple sites (Gundem et al. 2015). Those rare subpopulations of cells within tumor foci that reactivate AR through acquisition of mutations, copy number alterations, or synthesis of constitutively active AR splice variants will evade ADT (Watson et al. 2015). This concept is supported by a recent targeted sequencing study in which germ line, plasma, and tumor samples from ERG-positive patients were collected before, during, and after treatment with abiraterone acetate or enzalutamide. This longitudinal analysis identified AR amplification and the appearance of AR point mutations in response to therapy (Carreira et al. 2014). An additional mechanism of increased AR signaling in response to ADT is expression of truncated AR variants (Dehm et al. 2008). Expression of AR-Vs has been detected in primary

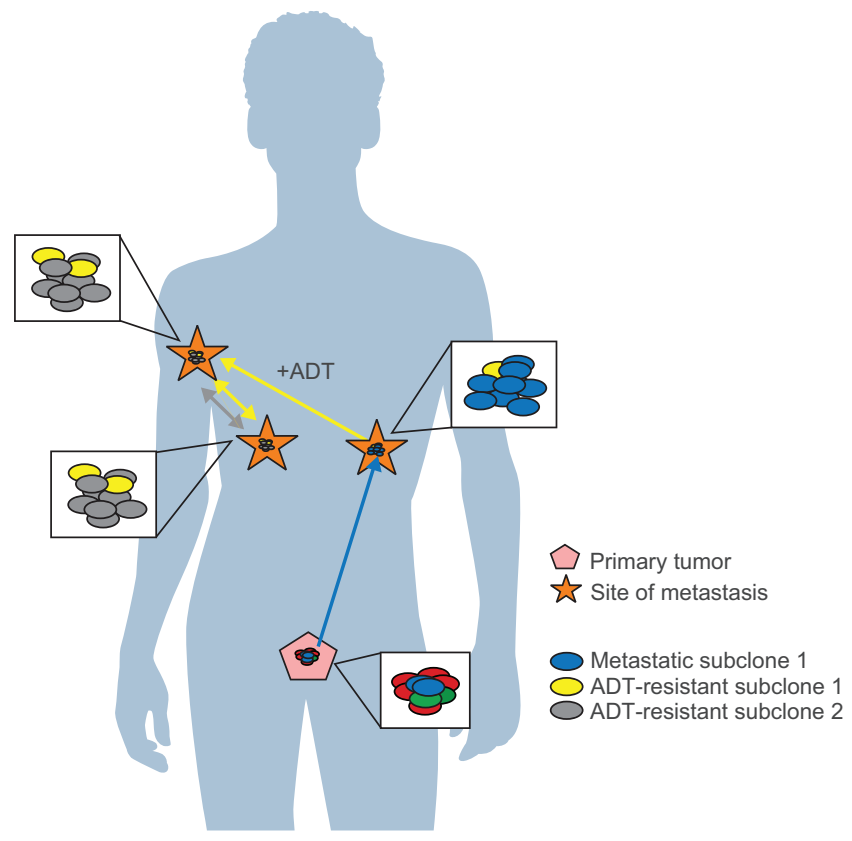

Figure 3

Treatment with hormone therapy selects and expands subclones harboring $A R$ amplification and mutations to confer treatment resistance. A rare tumor clone (blue, "metastatic subclone 1") acquires metastatic potential and seeds the falciform ligament (depicted by a blue arrow), where subclonal populations acquire new mutations. Upon treatment with $A D T$, a subclonal population harboring mutations that promote ADT resistance (yellow, "ADT-resistant subclone 1") undergoes clonal expansion and spreads to a site in the axillary lymph node (depicted by yellow arrows). Acquisition of new mutations also occurs to generate another subclonal population resistant to ADT (gray). The mixture of ADT-resistant clones seeds the R. rib in a polyclonal manner (depicted by yellow and gray arrows, adapted from Gundem et al. 2015).

tumors, metastases, as well as circulating tumor cells (Abeshouse et al. 2015). AR-Vs function as constitutively active transcription factors and can enable CRPC cells to escape AR-directed therapies that require the intact AR ligand binding domain (Dehm et al. 2008, Guo et al. 2009, Sun et al. 2010, Watson et al. 2010, Li et al. 2011, Hu et al. 2012). Intriguingly, RNA-seq studies have indicated that AR-V expression relative to full-length AR does not increase dramatically in metastatic CRPC tissues relative to primary tumor tissues (Abeshouse et al. 2015, Robinson et al. 2015). This finding suggests that subclonal expansion of AR-V-expressing cells does not occur in metastatic disease and may instead represent a property of rare populations of tumor cells that are resistant to ADT. However, these findings from RNA-seq-based studies are in contrast to RT-PCR-based studies, which have found higher expression of AR-Vs in CRPC tissues relative to primary prostate cancer, as well as correlations between AR-V expression and with poor outcomes ( $\mathrm{Hu}$ et al. 2009, Sun et al. 2010, Hornberg et al. 2011).

Published by Bioscientifica Ltd. 
One provocative observation in recent studies has been that the acquisition of various $A R$ mutation and amplification events at metastatic sites can occur through polyclonal seeding mechanisms (Fig. 3) (Gundem et al. 2015, Hong et al. 2015). For example, in one patient, two subclones involved in polyclonal seeding harbored two different alterations that promote ADT resistance, including MYC amplification and a pathogenic AR substitution (Gundem et al. 2015). Similarly, in a longitudinal biopsy study, a metastatic site in the iliac crest displayed profound clonal evolution in response to ADT, ultimately becoming enriched for a subpopulation of cells that had originated in a sacral metastasis (Hong et al. 2015). In patients that exhibited polyclonal seeding, several subclones across metastases were shared and were therefore more similar to each other than to the primary tumor (Gundem et al. 2015). Together, these findings suggest that interclonal cooperation may occur and that multiple independent ADT-resistant subclones may arise and ultimately cooperate to drive subclonal expansion in response to ADT. There is precedent for polyclonal seeding in murine models of mammary tumors; however, until recently, this has not been observed in human tissues (Cleary et al. 2014, Marusyk et al. 2014). For example, in WNT-driven mammary tumors, HRASmt WNT ${ }^{\text {low }}$ basal and HRAS ${ }^{\text {wt }}$ WNThigh luminal subclones cooperate interclonally to propagate tumor growth and response to WNT-targeted therapy (Cleary et al. 2014). In these cases, acquisition of multiple mutations across subclonal populations appears to promote and may be necessary to drive tumor growth and clonal expansion under treatment conditions.

\section{Clones representative of multiple stages of disease persist in blood}

Tumor cells representing various stages and sites of disease are detectable in blood. Thus, circulating tumor cells and cell-free circulating tumor DNA provide a window into the totality of disease within a patient. Recently, a number of studies have demonstrated detection of biomarkers of advanced disease and therapy resistance in patients with advanced prostate cancer. For example, a clone originating from a primary tumor was detected by targeted sequencing of genomic DNA isolated from blood 3 years after resection of the prostate (Hong et al. 2015). In the same patient, a metastatic clone that originated in the primary tumor but seeded a metastatic site in the seminal vesicle was detectable in blood (Hong et al. 2015). Targeted sequencing of DNA isolated from plasma revealed the emergence of T878A and $\mathrm{L} 702 \mathrm{H}$ mutations in the AR during progression on abiraterone (Romanel et al. 2015). In the same study, patients with AR amplification or $A R$ mutation were less likely to experience a PSA decline after initiation of therapy with abiraterone acetate. Importantly, the presence of mutant AR alleles was more common in patients that did not display evidence for $A R$ gene amplification, indicating that clones exhibiting single alterations in $A R$ may be sufficient to impart therapy resistance (Romanel et al. 2015). Accordingly, $A R$ amplification was detected in circulating cell-free DNA and was shown to be associated with enzalutamide and abiraterone treatment resistance in a cohort of 62 CRPC patients, albeit at a higher frequency in those treated with enzalutamide versus those treated with abiraterone acetate (Azad et al. 2015).

AR variants that are detectable at the mRNA level in circulating tumor cells are predictive of poor response to treatment with abiraterone acetate or enzalutamide (Antonarakis et al. 2014, 2015). In a cohort of patients with castration-resistant disease, more than 50\% of patients had detectable expression of at least one AR-V mRNA in single circulating tumor cells isolated from blood, and $\sim 17 \%$ expressed more than one AR-V in an individual circulating tumor cell (Miyamoto et al. 2015). In the same study, the authors observed alterations in GR and Wnt signaling in different subsets of circulating tumor cells. Variations in subclonal abundance were observed in patients with CRPC before, during, and after treatment with abiraterone (Carreira et al. 2014). In one patient, biopsy of a liver metastasis revealed a W742C mutation in $A R$, whereas circulating DNA revealed $A R$ amplification. Treatment with docetaxel resolved the liver metastasis; however, the patient exhibited no response to abiraterone acetate, as defined by a 50\% decline from baseline in serum PSA levels. This was likely due to the occult subclonal lesions harboring $A R$ amplification, which were detectable in plasma DNA (Carreira et al. 2014). Together, these studies indicate the presence of multiple subclonal populations within the circulation of a single patient. Overall, capture of circulating tumor cells or cell-free DNA has provided a useful approach to gain a snapshot of subclonal populations and their evolution during disease progression. As such, these studies have generated enthusiasm around the ultimate use of circulating tumor cell or cell-free DNA analysis to help inform treatment decisions for individual patients.

\section{Summary}

Retrospective and real-time computational renderings of the clonal architecture within patient primary tumors and

Published by Bioscientifica Ltd 
metastases have provided an unprecedented window into clonal evolution across progressive disease. A tremendous amount of genetic information is available describing the mutations, structural alterations, and altered pathways in both primary and metastatic prostate cancer, yet they are limited in that they can only provide genetic alterations across bulk tissue samples (Grasso et al. 2012, Abeshouse et al. 2015, Robinson et al. 2015). Here, we have reviewed studies that confirm the clonal heterogeneity of primary and metastatic prostate cancer and provide early evidence that metastatic spread can occur via several mechanisms and not one distinct linear pathway. Furthermore, the studies discussed here have provided the first insights into the temporal acquisition of gene alterations during metastasis and therapy resistance of prostate cancer.

A consensus finding from these studies is that loss or mutation of TP53 and PTEN loss occur before or early in metastasis, indicating that they drive metastatic spread. The recently identified mutually exclusive molecular subtypes of primary prostate cancer including ETS fusions and mutations in FOXA1, FLI1, SPOP, and IDH1 represent early drivers of tumorigenesis, and at least some molecular basis for inter-patient heterogeneity. However, acquisition of mutations in drivers of metastasis does not appear to occur preferentially in any one of these molecular subtypes, indicating that the transition to metastatic disease may follow a somewhat unified biological mechanism. Another key finding in these studies is that AR, which is altered in $>60 \%$ of metastatic prostate cancer and known to be a mediator of ADT resistance (reviewed in Watson et al. 2015), undergoes alterations after metastasis has occurred. This novel finding indicates that metastasis and therapy resistance are temporally separate processes. However, it remains unclear whether rare subclones originating in the primary tumor or early metastases harbor AR alterations that later promote ADT resistance or, alternatively, whether such alterations arise after metastasis and initial treatment with ADT. Further analysis of circulating tumor cells from patients at various stages of disease progression may help to understand whether resistant tumor clones are present before ADT.

In addition to providing insight into the temporal landscape of genetic alterations across prostate cancer development, the studies reviewed here have provided views of the mechanisms of metastatic spread. Foremost, these studies have established that metastatic spread can occur through monoclonal or polyclonal seeding between metastases or in waves originating from the primary tumor. Furthermore, they have demonstrated that clonal spread is not unidirectional, as metastatic clones can reseed the surgical bed of the resected prostate. The mechanistic underpinnings of metastasis-to-metastasis seeding remain to be understood. For example, the properties of the metastatic niche that are required for either monoclonal or polyclonal seeding or reseeding by metastatic clonal populations are largely unknown. Nevertheless, these studies have highlighted the complexity of patterns of clonal spread and subclonal evolution in response to environmental and therapeutic pressures such as ADT. Monitoring circulating tumor cells and circulating cell-free DNA provides an opportunity to track the totality of these complex disease processes within an individual patient and possibly identifies subclones harboring clinically actionable mutations or early indicators of therapeutic resistance.

Declaration of interest

S M D has served as a paid consultant/advisor for Astellas/Medivation.

\section{Funding}

S M D is currently funded by a Movember/Prostate Cancer Foundation Challenge Award; American Cancer Society Research Scholar Grant RSG-12031-01-TBE; NIH grant R01CA174777; US Department of Defense Prostate Cancer Research Program grants W81XWH-12-2-0093, W81XWH-13-1-0518, W81XWH-15-1-0633, and W81XWH-15-1-0501; and a grant from the Minnesota Partnership for Biotechnology and Medical Genomics. J L V is supported by the Cancer Biology Training Grant T32 CA009138.

\section{References}

Abeshouse A, Ahn J, Akbani R, Ally A, Amin S, Andry Christopher D, Annala M, Aprikian A, Armenia J, Arora A, et al. 2015 The molecular taxonomy of primary prostate cancer. Cell 163 1011-1025. (doi:10.1016/j.cell.2015.10.025)

Adamo P \& Ladomery MR 2015 The oncogene ERG: a key factor in prostate cancer. Oncogene 35 403-414. (doi:10.1038/onc.2015.109) Antonarakis ES, Lu C, Wang H, Luber B, Nakazawa M, Roeser JC, Chen Y, Mohammad TA, Chen Y, Fedor HL, et al. 2014 AR-V7 and resistance to enzalutamide and abiraterone in prostate cancer. New England Journal of Medicine 371 1028-1038. (doi:10.1056/ NEJMoa1315815)

Antonarakis ES, Lu C, Luber B, Wang H, Chen Y, Nakazawa M, Nadal R, Paller CJ, Denmeade SR, Carducci MA, et al. 2015 Androgen receptor splice variant 7 and efficacy of taxane chemotherapy in patients with metastatic castration-resistant prostate cancer. JAMA Oncology $\mathbf{1}$ 582-591. (doi:10.1001/jamaoncol.2015.1341)

Azad AA, Volik SV, Wyatt AW, Haegert A, Le Bihan S, Bell RH, Anderson SA, McConeghy B, Shukin R, Bazov J, et al. 2015 Androgen receptor gene aberrations in circulating cell-free DNA: biomarkers of therapeutic resistance in castration-resistant prostate cancer. Clinical Cancer Research 21 2315-2324. (doi:10.1158/1078-0432.CCR14-2666)

Baca SC, Prandi D, Lawrence MS, Mosquera JM, Romanel A, Drier Y, Park K, Kitabayashi N, MacDonald TY, Ghandi M, et al. 2013 Punctuated evolution of prostate cancer genomes. Cell 153 666-677. (doi:10.1016/j.cell.2013.03.021)

Barbieri CE, Baca SC, Lawrence MS, Demichelis F, Blattner M, Theurillat JP, White TA, Stojanov P, Van Allen E, Stransky N, et al.

Published by Bioscientifica Ltc 
2012 Exome sequencing identifies recurrent SPOP, FOXA1 and MED12 mutations in prostate cancer. Nature Genetics 44 685-689. (doi:10.1038/ng.2279)

Barbieri CE, Bangma CH, Bjartell A, Catto JW, Culig Z, Gronberg H, Luo J, Visakorpi T \& Rubin MA 2013 The mutational landscape of prostate cancer. European Urology 64 567-576. (doi:10.1016/j.eururo.2013.05.029)

Barry M, Perner S, Demichelis F \& Rubin MA 2007 TMPRSS2-ERG fusion heterogeneity in multifocal prostate cancer: clinical and biologic implications. Urology 70 630-633. (doi:10.1016/j.urology.2007.08.032)

Beer TM, Armstrong AJ, Rathkopf DE, Loriot Y, Sternberg CN, Higano CS, Iversen P, Bhattacharya S, Carles J, Chowdhury S, et al. 2014 Enzalutamide in metastatic prostate cancer before chemotherapy. New England Journal of Medicine 371 424-433. (doi:10.1056/ NEJMoa1405095)

Bolli N, Avet-Loiseau H, Wedge DC, Van Loo P, Alexandrov LB, Martincorena I, Dawson KJ, Iorio F, Nik-Zainal S, Bignell GR, et al. 2014 Heterogeneity of genomic evolution and mutational profiles in multiple myeloma. Nature Communications 5 2997. (doi:10.1038/ncomms3997)

Boutros PC, Fraser M, Harding NJ, de Borja R, Trudel D, Lalonde E, Meng A, Hennings-Yeomans PH, McPherson A, Sabelnykova VY, et al. 2015 Spatial genomic heterogeneity within localized, multifocal prostate cancer. Nature Genetics 47 736-745. (doi:10.1038/ng.3315)

Boyd LK, Mao X, Xue L, Lin D, Chaplin T, Kudahetti SC, Stankiewicz E, Yu Y, Beltran L, Shaw G, et al. 2012 High-resolution genome-wide copynumber analysis suggests a monoclonal origin of multifocal prostate cancer. Genes Chromosomes Cancer 51 579-589. (doi:10.1002/gcc.v51.6)

Campbell PJ, Pleasance ED, Stephens PJ, Dicks E, Rance R, Goodhead I, Follows GA, Green AR, Futreal PA \& Stratton MR 2008 Subclonal phylogenetic structures in cancer revealed by ultra-deep sequencing. PNAS 105 13081-13086. (doi:10.1073/pnas.0801523105)

Carreira S, Romanel A, Goodall J, Grist E, Ferraldeschi R, Miranda S, Prandi D, Lorente D, Frenel JS, Pezaro C, et al. 2014 Tumor clone dynamics in lethal prostate cancer. Science Translational Medicine 6 254ra125. (doi:10.1126/scitranslmed.3009448)

Cheng L, Song SY, Pretlow TG, Abdul-Karim FW, Kung HJ, Dawson DV, Park WS, Moon YW, Tsai ML, Linehan WM, et al. 1998 Evidence of independent origin of multiple tumors from patients with prostate cancer. Journal of the National Cancer Institute 90 233-237. (doi:10.1093/jnci/90.3.233)

Cleary AS, Leonard TL, Gestl SA \& Gunther EJ 2014 Tumour cell heterogeneity maintained by cooperating subclones in Wnt-driven mammary cancers. Nature 508 113-117. (doi:10.1038/nature13187)

Cooper CS, Eeles R, Wedge DC, Van Loo P, Gundem G, Alexandrov LB, Kremeyer B, Butler A, Lynch AG, Camacho N, et al. 2015 Analysis of the genetic phylogeny of multifocal prostate cancer identifies multiple independent clonal expansions in neoplastic and morphologically normal prostate tissue. Nature Genetics 47 367-372. (doi:10.1038/ng.3221)

Dehm SM \& Tindall DJ 2011 Alternatively spliced androgen receptor variants. Endocrine-Related Cancer 18 R183-R196. (doi:10.1530/ERC11-0141)

Dehm SM, Schmidt LJ, Heemers HV, Vessella RL \& Tindall DJ 2008 Splicing of a novel androgen receptor exon generates a constitutively active androgen receptor that mediates prostate cancer therapy resistance. Cancer Research 68 5469-5477. (doi:10.1158/0008-5472. CAN-08-0594)

Ding L, Ley TJ, Larson DE, Miller CA, Koboldt DC, Welch JS, Ritchey JK, Young MA, Lamprecht T, McLellan MD, et al. 2012 Clonal evolution in relapsed acute myeloid leukaemia revealed by whole-genome sequencing. Nature 481 506-510. (doi:10.1038/nature10738)

Grasso CS, Wu YM, Robinson DR, Cao X, Dhanasekaran SM, Khan AP, Quist MJ, Jing X, Lonigro RJ, Brenner JC, et al. 2012 The mutational landscape of lethal castration-resistant prostate cancer. Nature $\mathbf{4 8 7}$ 239-243. (doi:10.1038/nature11125)

Greaves M \& Maley CC 2012 Clonal evolution in cancer. Nature 481 306-313. (doi:10.1038/nature10762)
Gundem G, Van Loo P, Kremeyer B, Alexandrov LB, Tubio JM, Papaemmanuil E, Brewer DS, Kallio HM, Hognas G, Annala M, et al. 2015 The evolutionary history of lethal metastatic prostate cancer. Nature 520 353-357. (doi:10.1038/nature14347)

Guo Z, Yang X, Sun F, Jiang R, Linn DE, Chen H, Chen H, Kong X, Melamed J, Tepper CG, et al. 2009 A novel androgen receptor splice variant is up-regulated during prostate cancer progression and promotes androgen depletion-resistant growth. Cancer Research 69 2305-2313. (doi:10.1158/0008-5472.CAN-08-3795)

Haffner MC, Mosbruger T, Esopi DM, Fedor H, Heaphy CM, Walker DA, Adejola N, Gurel M, Hicks J, Meeker AK, et al. 2013 Tracking the clonal origin of lethal prostate cancer. Journal of Clinical Investigation 123 4918-4922. (doi:10.1172/JCI70354)

Haffner MC, Weier C, Xu MM, Vaghasia A, Gurel B, Gumuskaya B, Esopi DM, Fedor H, Tan HL, Kulac I, et al. 2015 Molecular evidence that invasive adenocarcinoma can mimic prostatic intraepithelial neoplasia (PIN) and intraductal carcinoma through retrograde glandular colonization. Journal of Pathology 238 31-41. (doi:10.1002/path.4628)

Hong MK, Macintyre G, Wedge DC, Van Loo P, Patel K, Lunke S, Alexandrov LB, Sloggett C, Cmero M, Marass F, et al. 2015 Tracking the origins and drivers of subclonal metastatic expansion in prostate cancer. Nature Communications 6 6605. (doi:10.1038/ncomms7605)

Hornberg E, Ylitalo EB, Crnalic S, Antti H, Stattin P, Widmark A, Bergh A \& Wikstrom P 2011 Expression of androgen receptor splice variants in prostate cancer bone metastases is associated with castration-resistance and short survival. PLoS One 6 e19059. (doi:10.1371/journal.pone.0019059)

Hu R, Dunn TA, Wei S, Isharwal S, Veltri RW, Humphreys E, Han M, Partin AW, Vessella RL, Isaacs WB, et al. 2009 Ligandindependent androgen receptor variants derived from splicing of cryptic exons signify hormone-refractory prostate cancer. Cancer Research 69 16-22. (doi:10.1158/0008-5472.CAN-08-2764)

$\mathrm{Hu}$ R, Lu C, Mostaghel EA, Yegnasubramanian S, Gurel M, Tannahill C, Edwards J, Isaacs WB, Nelson PS, Bluemn E, et al. 2012 Distinct transcriptional programs mediated by the ligand-dependent full-length androgen receptor and its splice variants in castration-resistant prostate cancer. Cancer Research 72 3457-3462. (doi:10.1158/0008-5472.CAN-11-3892)

Hugel A \& Wernert N 1999 Loss of heterozygosity (LOH), malignancy grade and clonality in microdissected prostate cancer. British Journal of Cancer 79 551-557. (doi:10.1038/sj.bjc.6690087)

Kim JH, Dhanasekaran SM, Mehra R, Tomlins SA, Gu W, Yu J, Kumar-Sinha C, Cao X, Dash A, Wang L, et al. 2007 Integrative analysis of genomic aberrations associated with prostate cancer progression. Cancer Research 67 8229-8239. (doi:10.1158/0008-5472. CAN-07-1297)

Knudsen KE \& Scher HI 2009 Starving the addiction: new opportunities for durable suppression of AR signaling in prostate cancer. Clinical Cancer Research 15 4792-4798. (doi:10.1158/1078-0432.CCR-08-2660)

Kobayashi M, Ishida H, Shindo T, Niwa S, Kino M, Kawamura K, Kamiya N, Imamoto T, Suzuki H, Hirokawa Y, et al. 2008 Molecular analysis of multifocal prostate cancer by comparative genomic hybridization. Prostate 68 1715-1724. (doi:10.1002/pros.v68:16)

Li Y, Alsagabi M, Fan D, Bova GS, Tewfik AH \& Dehm SM 2011 Intragenic rearrangement and altered RNA splicing of the androgen receptor in a cell-based model of prostate cancer progression. Cancer Research 71 2108-2117. (doi:10.1158/0008-5472.CAN-10-1998)

Lin C, Yang L, Tanasa B, Hutt K, Ju BG, Ohgi K, Zhang J, Rose DW, Fu XD, Glass CK, et al. 2009 Nuclear receptor-induced chromosomal proximity and DNA breaks underlie specific translocations in cancer. Cell 139 1069-1083. (doi:10.1016/j.cell.2009.11.030)

Lindberg J, Klevebring D, Liu W, Neiman M, Xu J, Wiklund P, Wiklund F, Mills IG, Egevad L \& Gronberg H 2013 Exome sequencing of prostate cancer supports the hypothesis of independent tumour origins. European Urology 63 347-353. (doi:10.1016/j.eururo.2012.03.050) 
Liu W, Laitinen S, Khan S, Vihinen M, Kowalski J, Yu G, Chen L, Ewing CM, Eisenberger MA, Carducci MA, et al. 2009 Copy number analysis indicates monoclonal origin of lethal metastatic prostate cancer. Nature Medicine 15 559-565. (doi:10.1038/nm.1944)

Lohr JG, Adalsteinsson VA, Cibulskis K, Choudhury AD, Rosenberg M, Cruz-Gordillo P, Francis JM, Zhang CZ, Shalek AK, Satija R, et al. 2014 Whole-exome sequencing of circulating tumor cells provides a window into metastatic prostate cancer. Nature Biotechnology $\mathbf{3 2}$ 479-484. (doi:10.1038/nbt.2892)

Mani RS, Tomlins SA, Callahan K, Ghosh A, Nyati MK, Varambally S, Palanisamy N \& Chinnaiyan AM 2009 Induced chromosomal proximity and gene fusions in prostate cancer. Science $\mathbf{3 2 6} 1230$. (doi:10.1126/science.1178124)

Marusyk A, Tabassum DP, Altrock PM, Almendro V, Michor F \& Polyak K 2014 Non-cell-autonomous driving of tumour growth supports subclonal heterogeneity. Nature $\mathbf{5 1 4} 54-58$. (doi:10.1038/nature13556)

McFadden DG, Papagiannakopoulos T, Taylor-Weiner A, Stewart C, Carter SL, Cibulskis K, Bhutkar A, McKenna A, Dooley A, Vernon A, et al. 2014 Genetic and clonal dissection of murine small cell lung carcinoma progression by genome sequencing. Cell 156 1298-1311. (doi:10.1016/j.cell.2014.02.031)

Merlo LM, Shah NA, Li X, Blount PL, Vaughan TL, Reid BJ \& Maley CC 2010 A comprehensive survey of clonal diversity measures in Barrett's esophagus as biomarkers of progression to esophageal adenocarcinoma. Cancer Prevention Research 3 1388-1397. (doi:10.1158/1940-6207.CAPR-10-0108)

Miyamoto DT, Zheng Y, Wittner BS, Lee RJ, Zhu H, Broderick KT, Desai R, Fox DB, Brannigan BW, Trautwein J, et al. 2015 RNA-Seq of single prostate CTCs implicates noncanonical Wnt signaling in antiandrogen resistance. Science 349 1351-1356. (doi:10.1126/ science.aab0917)

Nik-Zainal S, Van Loo P, Wedge DC, Alexandrov LB, Greenman CD, Lau KW, Raine K, Jones D, Marshall J, Ramakrishna M, et al. 2012 The life history of 21 breast cancers. Cell 149 994-1007. (doi:10.1016/j.cell.2012.04.023)

Nowell PC 1976 The clonal evolution of tumor cell populations. Science 194 23-28. (doi:10.1126/science.959840)

Rashid NU, Sperling AS, Bolli N, Wedge DC, Van Loo P, Tai YT, Shammas MA, Fulciniti M, Samur MK, Richardson PG, et al. 2014 Differential and limited expression of mutant alleles in multiple myeloma. Blood 124 3110-3117. (doi:10.1182/blood-2014-04569327)

Robbins CM, Tembe WA, Baker A, Sinari S, Moses TY, BeckstromSternberg S, Beckstrom-Sternberg J, Barrett M, Long J, Chinnaiyan A, et al. 2011 Copy number and targeted mutational analysis reveals novel somatic events in metastatic prostate tumors. Genome Research 21 47-55. (doi:10.1101/gr.107961.110)

Robinson D, Van Allen EM, Wu YM, Schultz N, Lonigro RJ, Mosquera JM, Montgomery B, Taplin ME, Pritchard CC, Attard G, et al. 2015 Integrative clinical genomics of advanced prostate cancer. Cell 161 1215-1228. (doi:10.1016/j.cell.2015.05.001)

Romanel A, Tandefelt DG, Conteduca V, Jayaram A, Casiraghi N, Wetterskog D, Salvi S, Amadori D, Zafeiriou Z, Rescigno P, et al. 2015 Plasma AR and abiraterone-resistant prostate cancer. Science Translational Medicine 7 312re310. (doi:10.1126/scitranslmed. aac9511)

Roudier MP, True LD, Higano CS, Vesselle H, Ellis W, Lange P \& Vessella RL 2003 Phenotypic heterogeneity of end-stage prostate carcinoma metastatic to bone. Human Pathology 34 646-653. (doi:10.1016/S0046-8177(03)00190-4)

Ruijter ET, Miller GJ, van de Kaa CA, van Bokhoven A, Bussemakers MJ, Debruyne FM, Ruiter DJ \& Schalken JA 1999 Molecular analysis of multifocal prostate cancer lesions. Journal of Pathology 188 271-277. (doi:10.1002/(sici)1096-9896(199907)188:3<271::aidpath359>3.0.co;2-k

Ryan CJ, Smith MR, de Bono JS, Molina A, Logothetis CJ, de Souza P, Fizazi K, Mainwaring P, Piulats JM, Ng S, et al. 2013 Abiraterone in metastatic prostate cancer without previous chemotherapy. New England Journal of Medicine 368 138-148. (doi:10.1056/ NEJMoa1209096)

Shah RB, Mehra R, Chinnaiyan AM, Shen R, Ghosh D, Zhou M, Macvicar GR, Varambally S, Harwood J, Bismar TA, et al. 2004 Androgen-independent prostate cancer is a heterogeneous group of diseases: lessons from a rapid autopsy program. Cancer Research 64 9209-9216. (doi:10.1158/0008-5472.CAN-04-2442)

Stephens PJ, Greenman CD, Fu B, Yang F, Bignell GR, Mudie LJ, Pleasance ED, Lau KW, Beare D, Stebbings LA, et al. 2011 Massive genomic rearrangement acquired in a single catastrophic event during cancer development. Cell 144 27-40. (doi:10.1016/j. cell.2010.11.055)

Sun S, Sprenger CC, Vessella RL, Haugk K, Soriano K, Mostaghel EA, Page ST, Coleman IM, Nguyen HM, Sun H, et al. 2010 Castration resistance in human prostate cancer is conferred by a frequently occurring androgen receptor splice variant. Journal of Clinical Investigation 120 2715-2730. (doi:10.1172/JCI41824)

Taylor BS, Schultz N, Hieronymus H, Gopalan A, Xiao Y, Carver BS, Arora VK, Kaushik P, Cerami E, Reva B, et al. 2010 Integrative genomic profiling of human prostate cancer. Cancer Cell 18 11-22. (doi:10.1016/j.ccr.2010.05.026)

Torre LA, Bray F, Siegel RL, Ferlay J, Lortet-Tieulent J \& Jemal A 2015 Global cancer statistics, 2012. CA: A Cancer Journal for Clinicians $\mathbf{6 5}$ 87-108. (doi:10.3322/caac.21262)

Van Loo P, Nordgard SH, Lingjaerde OC, Russnes HG, Rye IH, Sun W, Weigman VJ, Marynen P, Zetterberg A, Naume B, et al. 2010 Allelespecific copy number analysis of tumors. PNAS 107 16910-16915. (doi:10.1073/pnas.1009843107)

Villers A, McNeal JE, Freiha FS \& Stamey TA 1992 Multiple cancers in the prostate. Morphologic features of clinically recognized versus incidental tumors. Cancer 70 2313-2318. (doi:10.1002/10970142(19921101)70:9<2313::aid-cncr2820700917>3.0.co;2-t)

Wan L, Pantel K \& Kang Y 2013 Tumor metastasis: moving new biological insights into the clinic. Nature Medicine 19 1450-1464. (doi:10.1038/nm.3391)

Watson PA, Chen YF, Balbas MD, Wongvipat J, Socci ND, Viale A, Kim K \& Sawyers CL 2010 Constitutively active androgen receptor splice variants expressed in castration-resistant prostate cancer require fulllength androgen receptor. PNAS 107 16759-16765. (doi:10.1073/ pnas.1012443107)

Watson PA, Arora VK \& Sawyers CL 2015 Emerging mechanisms of resistance to androgen receptor inhibitors in prostate cancer. Nature Reviews Cancer 15 701-711. (doi:10.1038/nrc4016)

Weischenfeldt J, Simon R, Feuerbach L, Schlangen K, Weichenhan D, Minner S, Wuttig D, Warnatz HJ, Stehr H, Rausch T, et al. 2013 Integrative genomic analyses reveal an androgen-driven somatic alteration landscape in early-onset prostate cancer. Cancer Cell $\mathbf{2 3}$ 159-170. (doi:10.1016/j.ccr.2013.01.002)

Received in final form 4 March 2016

Accepted 17 March 2016

Accepted Preprint published online 21 March 2016 http://erc.endocrinology-journals.org DOI: 10.1530/ERC-16-0049
(C) 2016 Society for Endocrinology Printed in Great Britain
Published by Bioscientifica Ltd 